\title{
The prognostic analysis of lung cancer patients with occult malignant pleural disease at thoracotomy
}

\author{
Shaolei Li ${ }^{1 \#}$, Xin Yang ${ }^{2 \#}$, Shanyuan Zhang ${ }^{1}$, Miao Huang ${ }^{1}$, Yuanyuan $\mathrm{Ma}^{1}$, Yue Yang ${ }^{1}$ \\ ${ }^{1}$ Department of Thoracic Surgery II, ${ }^{2}$ Department of Pathology, Key Laboratory of Carcinogenesis and Translational Research (Ministry of \\ Education), Peking University Cancer Hospital and Institute, Beijing 100142, China \\ Contributions: (I) Conception and design: S Li, X Yang, Y Yang; (II) Administrative support: None; (III) Provision of study materials or patients: \\ None; (IV) Collection and assembly of data: M Huang, S Zhang; (V) Data analysis and interpretation: Y Ma; (VI) Manuscript writing: All authors; (VII) \\ Final approval of manuscript: All authors. \\ "These authors contributed equally to this work. \\ Correspondence to: Yue Yang. Department of Thoracic Surgery II, Peking University Cancer Hospital \& Institute, 52 Fucheng Road, Haidian District, \\ Beijing 100142, China. Email: zlyangyue@bjmu.edu.cn.
}

Background: This study aims to determine the clinicopathological prognostic factors for occult malignant pleural disease (MPD) that were first detected in patients with non-small cell lung cancer (NSCLC) at thoracotomy and to assess the outcome of surgical intervention.

Methods: A total of 120 thoracotomy-patients with MPD were examined. The Kaplan-Meier test estimated survival curves, and Cox regression analysis was performed to validate the risk factors picked. In assessing surgical intervention, clinical and pathological parameters were matched by propensity score matching (PSM). Results: With a median follow-up of 34 months, the 5 -year overall survival of 120 patients was $28.0 \%$. Multivariate analyses showed male $(\mathrm{P}=0.044)$, advanced $\mathrm{T}$ stages $(\mathrm{P}<0.001)$, advanced $\mathrm{N}$ stages $(\mathrm{P}=0.02)$, pleural invasion in image $(\mathrm{P}=0.005)$, pleural effusion $(\mathrm{P}=0.027)$, surgical intervention $(\mathrm{P}=0.008)$ and EGFR status $(\mathrm{P}=0.003)$ were independent predictors of survival. The 5 -year survival rate and median survival time (MST) for 21 patients with lobectomy and pleurectomy were $71.6 \%$ and undefined, compared with $25.6 \%$ and 40.0 months in 46 patients with sublobectomy. When 53 patients only subjected to open-close surgery, their 5-year survival rate and MST were 23.4\% and 30.2 months. After PSM, both 21 patients were included in lobectomy with the pleurectomy group and sublobectomy /open-close group. The overall survival of lobectomy with the pleurectomy group was better than the control group $(\mathrm{P}=0.046)$.

Conclusions: Age, stage, pleural invasion, pleural effusion, surgical intervention, and EGFR status affected the prognosis of the MPD patients first diagnosed at thoracotomy. Selective surgery gives better recovery, and additional studies are needed.

Keywords: Malignant pleural disease (MPD); lung cancer; EGFR; surgery

Submitted Aug 31, 2019. Accepted for publication Jan 18, 2020.

doi: $10.21037 /$ tcr.2020.02.03

View this article at: http://dx.doi.org/10.21037/tcr.2020.02.03 


\section{Introduction}

The latest edition of the TNM classification for non-small cell lung cancer (NSCLC) affirmed the importance of the previously existing M1a descriptor definition featuring patients in this group with pleural/pericardial effusions (PE), contralateral or bilateral lung nodules, or pleural/pericardial nodules (PNs). There were no significant differences observed between the various groups set out in this M1a descriptor definition with poor prognosis as median survival time (MST) 11.5 months (1). However, occult malignant pleural diseases (MPD), including PN with/without PE, were occasionally only discovered at thoracotomy with relatively better outcomes as MST 34.0 months, and then the decision whether to remove or not remove the primary tumor lesion is regularly surrounded by controversy (2). The main tumor and visible pleural nodule resection were usually performed in minimal disease with less tumor burden, and open-close surgery was more likely to perform in advanced disease with multiple pleural nodules and pleural effusion, which was the primary selection bias in previous studies (3). This study's purpose was to ascertain the clinical and biological prognostic factors for occult MPD initially identified by thoracotomy and evaluate the results of surgical intervention using propensity score matching (PSM) analysis.

\section{Methods}

\section{Patients}

The subjects of this study were NSCLC patients who underwent surgery at the Department of Thoracic Surgery II, Peking University Cancer Hospital from January 2006 to October 2016. The 2,894 consecutive patients who had histologic diagnoses of NSCLC and received thoracotomy or video-assisted thoracic surgery were included in this study.

Computed tomography (CT) thorax scan, a full bone scan, a CT or magnetic resonance imaging (MRI) scan of the brain, and ultrasound scanning of the entire body's abdomen and supraclavicular lymph nodes or positron emission tomography (PET)/CT assisted in ruling out remote metastases. Endobronchial ultrasoundguided transbronchial needle aspiration (EBUS-TBNA), transesophageal endoscopic ultrasound-guided fineneedle aspiration (EUS-FNA) or mediastinoscopy were used to identify preoperative N2-stage patients. All the patients underwent full oncological staging before surgery, excluding clinical stage IV disease. However, among them, 123 patients $(4.25 \%)$ were proven with pathological M1a disease based on the most recent (eighth edition) UICC staging system at the time the surgery was carried out, who had MPD including PN without or with PE. Three patients had incomplete clinical data. Therefore, we analyzed 120 patients in this study. We named the situation of those 120 patients as occult MPD.

Signed informed consent was collected from all patients before thoracotomy, including a statement of approval for their disease information that future retrospective studies could use. Approval for this study was granted by the Ethics Committee of Peking University Cancer Hospital.

\section{Surgical procedures}

All the thoracotomy and surgical resections were carried out by surgeons based at the Department of Thoracic Surgery II of Peking University Cancer Hospital. One hundred twenty individuals with occult MPD were classified into three groups based on different surgical interventions. As many as 53 patients only experienced exploratory thoracotomy with biopsy of metastasis focuses, which was defined as openclose surgery, and the other 67 patients experienced primary lesion resection including 46 sublobectomy with/without pleurectomy and 21 lobectomies with pleurectomy. No matter whether the primary tumor lesion was resected, we systematically sampled the lymph nodes for more precise $\mathrm{N}$ stages.

\section{Statistical analysis}

Our analysis explored the clinicopathological attributes, therapy statuses and survival outcomes of occult MPD patients. Survival was estimated by the life table method in different groups, including gender, age, smoking history, drinking history, neoadjuvant therapy, PET/CT scan, tumor location, T stage, $\mathrm{N}$ stage, pathological type, pleural invasion in the image, vascular thrombosis, and EGFR status. Multivariate analysis was carried out by applying the Cox regression (backward stepwise) method. Followup was taken from our outpatient files and statistical office. One September 2018 served as the follow-up cutoff date. The Kaplan-Meier method was applied to draw up estimates for survival curves, and the log-rank test assessed significances. The terminal event was death from any cause. Matching of individual propensity scores was carried out with the nearest-neighbor method, with no 
replacement, and 0.1-caliper width. Ultimately, 21 patients from the lobectomy group and 21 matched patients from the sublobectomy/open-close group were involved in our analysis. Comparisons between the attributes of patients in the two groups took place prior to and following propensity scores being matched. Statistical significance was considered to exist when $\mathrm{P}<0.05$. Each statistical comparison was calculated with the SPSS Statistics 22.0 software package (SPSS Inc.).

\section{Results}

There were 123 patients ( $4.25 \%$ ) patients with MPD observed at thoracotomy. Of available for analysis 120 patients, 99 had preoperatively pleural invasions in CT images, which were defined as clinical stage T2a. Seventyeight patients had only PN without PE. Forty-two patients had a light $\mathrm{PE}$, including malignant or reactive $\mathrm{PE}(<100$ $\mathrm{mL}$ ) with $\mathrm{PN}$ diagnosed at thoracotomy which was occasionally suspected before surgery. There were 110 adenocarcinoma, 6 squamous carcinomas, 1 adenosquamous carcinoma, 2 large cell carcinoma and one other carcinoma. Of 120 MPD patients, 32 had EGFR mutation, 25 had wild-type tumors and the other 63 had unknown EGFR status. Table 1 sets out patients' characteristics.

The MST and 5-year survival rate of the 120 patients were, collectively, 34 months and $28 \%$, respectively. We estimated survival among distinct groups by KaplanMeier method, and the results were also presented in Table 1 including MST and 5-year survival. Differences were statistically significant in different gender $(\mathrm{P}=0.008)$, smoking status $(\mathrm{P}=0.03)$, $\mathrm{T}$ stages $(\mathrm{P}<0.001)$, pleural invasion in image $(\mathrm{P}=0.022)$, pleural effusion $(\mathrm{P}=0.003)$, surgical interventions $(\mathrm{P}=0.02)$, pathological types $(\mathrm{P}=0.02)$ and EGFR status $(\mathrm{P}=0.001)$. Except for those variables, drinking status $(\mathrm{P}=0.057), \mathrm{N}$ stages $(\mathrm{P}=0.079)$ and CEA levels $(\mathrm{P}=0.089)$ were also incorporated into multivariate analyses by applying the Cox proportional hazards model (Table 2). Male (HR 1.693, 95\% CI: 1.015-2.826, $\mathrm{P}=0.044$ ), advanced T stages (HR 3.937, 95\% CI: 2.028-7.642, $\mathrm{P}<0.001$ ), advanced $\mathrm{N}$ stages (HR 1.855, 95\% CI: $1.104-$ $3.116, \mathrm{P}=0.02$ ), pleural invasion in image (HR $3.188,95 \%$ CI: $1.415-7.181, \mathrm{P}=0.005)$, pleural effusion (HR 1.77, $95 \%$ CI: 1.067-2.936, $\mathrm{P}=0.027$ ), surgical intervention (open-close vs. lobectomy HR 3.688, 95\% CI: 1.409-9.655, $\mathrm{P}=0.008$ / sublobectomy $v s$. lobectomy HR 3.275, 95\% CI: $1.231-$ 8.710, $\mathrm{P}=0.017$ ) and EGFR status (HR 4.164, 95\% CI: $1.645-10.541, \mathrm{P}=0.003)$ persisted as independent predictors of survival.

Ninety-nine patients with pleural invasion in the image had worse prognosis with 5-year survival $24.3 \%$ and MST 38.3 months. In comparison, the 5 -year survival of the 21 patients without the pleural invasion in the image was $55.9 \%$, and the MST had not been achieved $(\mathrm{P}=0.022$, Figure 1A). Seventy-eight $\mathrm{PN}$ patients without $\mathrm{PE}$ had a superior prognosis, with a 5 -year survival rate of $37.6 \%$ and MST of 49.3 months, compared to $42 \mathrm{PN}$ patients with PE, with a 5-year survival rate of $14.2 \%$ and MST of 34.7 months $(\mathrm{P}=0.003$, Figure $1 B)$. The EGFR mutation meant the patients could receive help from EGFR-TKI therapy. Thus the 32 patients with EGFR mutation had an exciting 72.7\% 5-year survival rate and not reached MST. Furthermore, the 25 wild-type patients and 63 patients with unknown EGFR status had a 5-year survival rate of $23.0 \%$ and MST of 41.4 months $(\mathrm{P}=0.001$, Figure $1 C)$.

Twenty-one patients who underwent lobectomy had a 5 -year survival rate of $71.6 \%$, and the MST had not been achieved, in contrast with $25.6 \%$ and 40.0 months in 46 patients with sublobectomy $(\mathrm{P}=0.037)$. For 53 patients who received exploratory thoracotomy (open-close surgery), the 5-year survival rate and MST were $23.4 \%$ and 30.2 months, respectively $(\mathrm{P}=0.006$, compared with lobectomy). No significant prognostic difference was observed when sublobectomy and open-close surgery were compared $(\mathrm{P}=0.255)$ (Figure 1D).

When PSM was carried out, both 21 matched cases from the lobectomy group and sublobectomy/open-close group were subjected to comparison and analysis. Table 3 sets out the clinicopathological attributes of the two groups prior to and following PSM. The two groups were well balanced in terms of baseline characteristics, including sex, age, T stage, $\mathrm{N}$ stage, pleural invasion in the image, and pleural effusion after propensity scores were matched. Figure 2 showed that the five-year survival rate and MST of the 21 patients with lobectomy were significantly better than those in the control group after PSM $(\mathrm{P}=0.046)$. The 5-year survival rate of the lobectomy group was $71.6 \%$, and the MST was not achieved. In comparison, the 5 -year survival rate of the control group was $40.8 \%$, and the MST was 37.0 months.

\section{Discussion}

The seventh edition of the Union for International Cancer Control (UICC) oversaw the reclassification of patients suffering from MPD, which includes malignant pleural effusion and/or malignant pleural nodules, from having 
Table 1 Characteristics of 120 occult MPD patients and median survival time (MST) and 5-year survival rate of a different subgroup

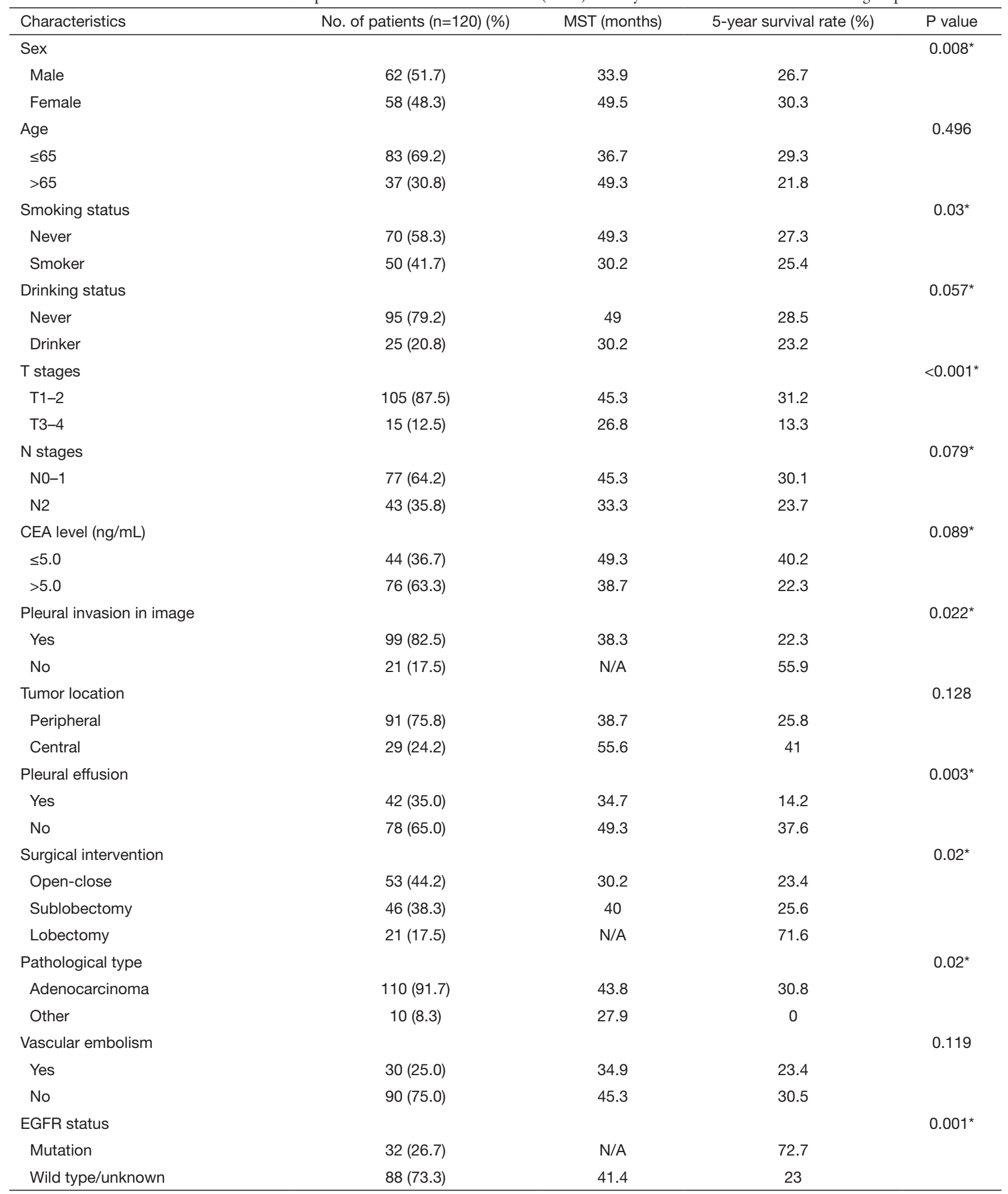

${ }^{*}$, the clinicopathological features of these $\mathrm{P}$ values were included in further multivariate analysis. MPD, malignant pleural disease. 
Table 2 Multivariate analysis of prognostic factors of 120 MPD patients using the Cox proportional hazards model

\begin{tabular}{llll}
\hline Variable & HR & 95\% Cl & P value \\
\hline Male & 1.693 & $1.015-2.826$ & 0.044 \\
T3-4 vs. T1-2 & 3.937 & $2.028-7.642$ & 0.001 \\
N2 vs. N0-1 & 1.855 & $1.104-3.116$ & 0.02 \\
Pleural invasion in image & 3.188 & $1.415-7.181$ & 0.005 \\
Pleural effusion & 1.77 & $1.067-2.936$ & 0.027 \\
Surgical intervention & & & 0.008 \\
Open-close vs. lobectomy & 3.688 & $1.409-9.655$ \\
Sublobectomy vs. lobectomy & 3.275 & $1.231-8.710$ \\
EGFR wild type/unknown & 4.164 & $1.645-10.541$ \\
\hline
\end{tabular}

MPD, malignant pleural disease.

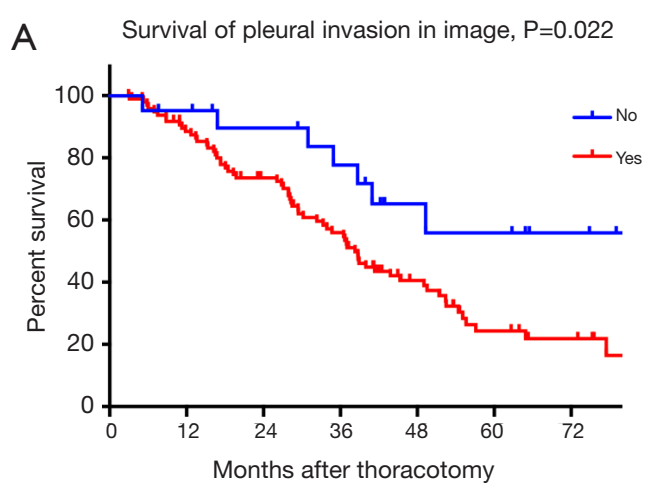

C

C Survival of EGFR status, $\mathrm{P}=0.001$

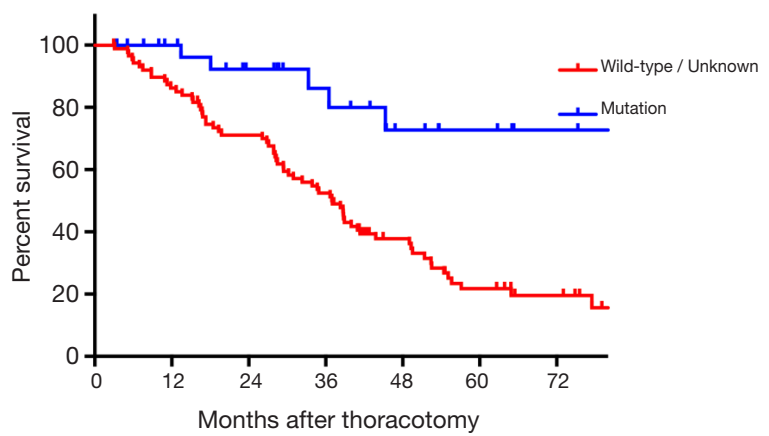

B Survival of pleural effusion, $P=0.003$

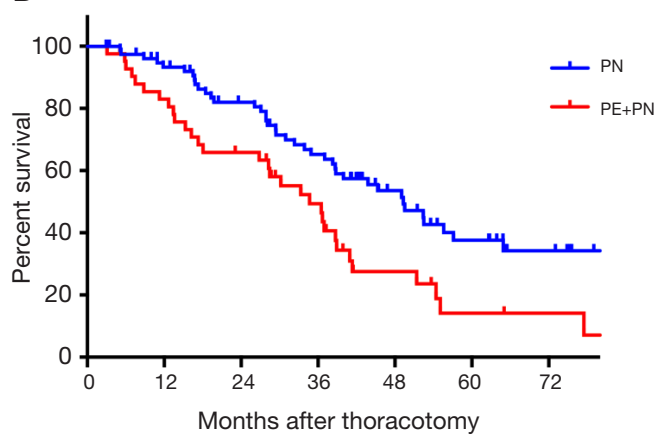

D Survival of surgical intervention, $P=0.02$

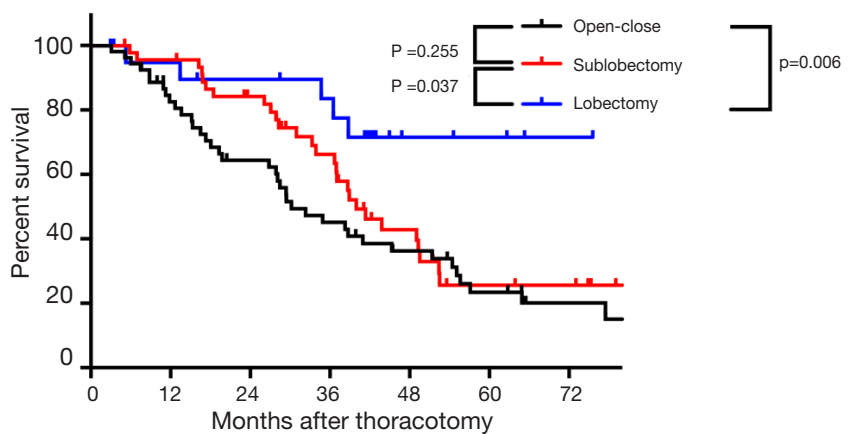

Figure 1 Curves of univariate survival analysis by Kaplan-Meier method. (A) Ninety-nine patients with pleural invasion in the image had a worse prognosis compared with 21 patients without pleural invasion. (B) Seventy-eight PN patients without PE had a significantly better prognosis than $42 \mathrm{PN}$ patients with PE. (C) Thirty-two patients with EGFR mutation had an exciting prognosis compared to the other 88 patients with wild type/unknown EGFR status. (D) The prognosis of 21 patients with lobectomy and pleurectomy was better than 46 patients subjected to sublobectomy and 53 patients with open-close surgery, but no significant prognostic difference was found when sublobectomy was compared with the open-close surgery. PN, pericardial nodule. 
Table 3 Characteristics of 120 occult MPD patients before and after propensity score matching

\begin{tabular}{|c|c|c|c|c|c|c|}
\hline Characteristics & \multicolumn{3}{|c|}{ Total cases } & \multicolumn{3}{|c|}{ Matched group } \\
\hline Male & $10(47.6)$ & $52(52.5)$ & & $10(47.6)$ & $9(42.9)$ & \\
\hline$\leq 65$ & $19(90.5)$ & $64(64.6)$ & & $19(90.5)$ & $19(90.5)$ & \\
\hline$>65$ & $2(9.5)$ & $35(35.4)$ & & $2(9.5)$ & $2(9.5)$ & \\
\hline T stages & & & 0.318 & & & 0.378 \\
\hline No-1 & $10(47.6)$ & $67(67.7)$ & & $10(47.6)$ & $10(47.6)$ & \\
\hline N2 & $11(52.4)$ & $32(32.3)$ & & $11(52.4)$ & $11(52.4)$ & \\
\hline \multicolumn{2}{|c|}{ Pleural invasion in image } & & 0.67 & & & 0.679 \\
\hline Yes & $18(85.7)$ & $81(81.8)$ & & $18(85.7)$ & $17(81.0)$ & \\
\hline No & $3(14.3)$ & $18(18.2)$ & & $3(14.3)$ & $4(19.0)$ & \\
\hline Pleural effusion & & & 0.092 & & & 1 \\
\hline Yes & $4(19.0)$ & $38(38.4)$ & & $4(19.0)$ & $4(19.0)$ & \\
\hline
\end{tabular}

MPD, malignant pleural disease.

stage IIIB (T4) to having stage IV (M1a) lung cancer (4). The classification of the 8th TNM allows the use of the existing category M1a. The IASLC Lung Cancer Staging Program indicated that 11.5 months of MST in patients with clinical MPD had a poor prognosis. While clinically diagnosed patients with MPD typically have a notable amount of malignant pleural effusion (5), MPD when observed with thoracotomy generally sees a lower amount of effusion or fewer pleural nodules, all of which have not been identified thorough pre-operative examination (6). Therefore, the progression of the disease in the pleural cavity among a subgroup of patients who have occult MPD could be at an earlier stage and as such, they may have a better prognosis than other M1 patients. The current study revealed an MST of 34 months as well as a 5-year survival rate of $28.0 \%$. Furthermore, we analyzed prognostic factors for occult MPD initially identified by thoracotomy and found gender, advanced $\mathrm{T}$ stages, advanced $\mathrm{N}$ stages, pleural invasion in the image, pleural effusion, surgery intervention, and EGFR status to be independent predictors of survival.

Visceral pleura invasion (VPI) is mainly a pathological descriptor and confers a worse prognosis $(7,8)$. At clinical staging, VPI can be assumed by the location of the tumor in relation to the surface of the lung and by its retraction, and despite the possibility of pathological confirmation (by true-cut biopsy, thoracoscopic biopsy, or wedge resection of tumor mass), it is not often confirmed, as it is inessential in the course of planning therapy. VPI is a key factor in prognosis and has been integrated into the latest IASLC lung cancer staging project (9). In this study, pleural invasion in CT image is also an independent prognostic factor in M1a patients, because it was the clinical feature of VPI. On the other hand, the patients with VPI were more easily suffered the MPD with/without pleural effusion $(10,11)$. Our study also revealed malignant pleural nodule (MPN) patients without PE had a better prognosis 


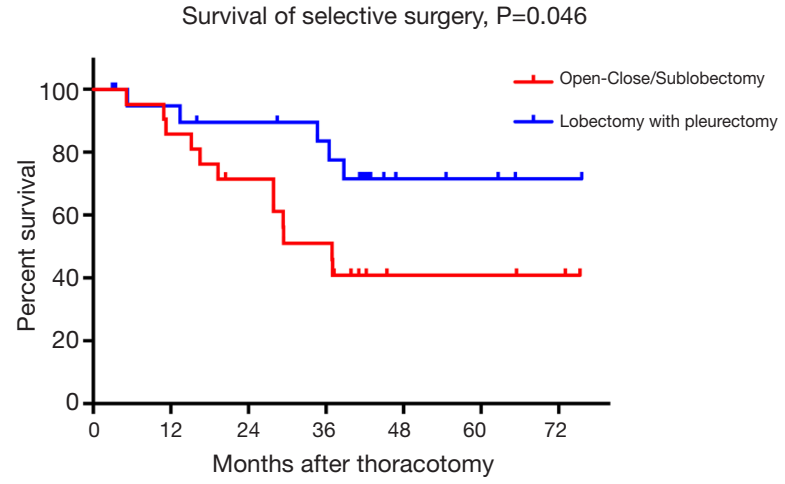

Figure 2 Following propensity score matching, 21 patients were included in lobectomy with the pleurectomy group and sublobectomy/open-close group. Overall survival of lobectomy was better for patients in the pleurectomy group than for their control group counterparts.

compared to MPN patients with PE, which was similar to the study of Okamoto et al. (12).

The incidence of occult MPD was ranged from 1.5-6.2\%, according to earlier studies. Although the pulmonary resection was associated with better survival in selected patients with MPD, no current guidelines support surgery over conservative therapy and the previous studies were not strong enough to change this recommendation (1222). It remained controversial whether surgery could ever be justified in NSCLC patients with an unexpected MPD at the surgery. Our results showed lobectomy with pleurectomy conferred to a better 5 -year survival rate with $71.6 \%$ and MST with 62.6 months compared to sublobectomy and open-close surgery. However, no significant difference was found to exist between the prognostic abilities of sublobectomy and open-close surgery. There may be some reviewers who questioned our results and thought choosing the method of surgical intervention was not random, and those patients in good health, young, less severe disease was chosen to do lobectomy with pleurectomy. Therefore, we conducted a bias matching score analysis for lobectomy and sublobectomy/open-close surgery and found that the long-term survival of lobectomy was better than that of patients without lobectomy. Given the previous review $(23,24)$ and this study, we concluded that lobectomy with pleurectomy or resection of greater range (such as extrapleural pneumonectomy) could improve the survival of occult MPD patients.

Recent data suggest that EGFR-TKIs (as opposed to standard first-line chemotherapy) should be used as first- line systemic treatment for patients in whom sensitizing EGFR mutations are recorded prior to first-line therapy (25-27). Our study showed that 32 patients with EGFR mutation had an exciting 5 -year survival rate of $72.7 \%$ and had not reached MST. In contrast, 25 wide-type patients and 63 unknown of EGFR status patients had poor survival who had no chance to accept EGFR-TKIs therapy.

Of course, a number of limitations existed in our study. First, as a surgical-case study conducted retrospectively, the subjects of this analysis were specifically selected and did not represent all patients with pleural carcinomatosis. Second, this study duration ranged from 2006 to 2016; 53 adenocarcinoma patients had unknown EGFR mutation status and no chance to receive help from targeted therapy. Because of these limitations and the promising survival in selected MPD patients, the future study will explore to regard occult MPD patients as a subgroup of M1a and design a randomized controlled trial to explore whether surgical resection should be performed.

In sum, our data prove gender, $\mathrm{T}$ stages, $\mathrm{N}$ stages, pleural invasion in the image, pleural effusion, surgery intervention, and EGFR status were independent predictors of survival of occult MPD. Selective surgery such as lobectomy with pleurectomy confers better prognosis and randomized controlled studies are needed in the future to explore the value of the surgical intervention.

\section{Acknowledgments}

We appreciate the help of the Chi-Li Pao Foundation at Stanford Medicine. The abstract for this manuscript was published in the Fournal of Thoracic Oncology October 2018 Volume 13, Issue 10, Supplement, Page S691 P2.01-67.

Funding: This work was supported by National Key R\&D Program of China (No.2018YFC0910700), National Natural Science Foundation of China (No. 81772494) and the Beijing Municipal Administration of Hospital's Youth Programme (No. QMS20191107).

\section{Footnote}

Conflicts of Interest: All authors have completed the ICMJE uniform disclosure form (available at http://dx.doi. org/10.21037/tcr.2020.02.03). The authors have no conflicts of interest to declare.

Etbical Statement: The authors are accountable for all aspects of the work in ensuring that questions related 
to the accuracy or integrity of any part of the work are appropriately investigated and resolved. The study was conducted in accordance with the Declaration of Helsinki (as revised in 2013). The Ethics Committee approved this study of Peking University Cancer Hospital. Signed informed consent was collected from all patients before thoracotomy, including a statement of approval for their disease information that future retrospective studies could use.

Open Access Statement: This is an Open Access article distributed in accordance with the Creative Commons Attribution-NonCommercial-NoDerivs 4.0 International License (CC BY-NC-ND 4.0), which permits the noncommercial replication and distribution of the article with the strict proviso that no changes or edits are made and the original work is properly cited (including links to both the formal publication through the relevant DOI and the license). See: https://creativecommons.org/licenses/by-nc-nd/4.0/.

\section{References}

1. Eberhardt WE, Mitchell A, Crowley J, et al. The IASLC Lung Cancer Staging Project: Proposals for the Revision of the $M$ Descriptors in the Forthcoming Eighth Edition of the TNM Classification of Lung Cancer. J Thorac Oncol 2015;10:1515-22.

2. Iida T, Shiba M, Yoshino I, et al. Surgical Intervention for Non-Small-Cell Lung Cancer Patients with Pleural Carcinomatosis: Results From the Japanese Lung Cancer Registry in 2004. J Thorac Oncol 2015;10:1076-82.

3. Li C, Kuo SW, Hsu HH, et al. Lung adenocarcinoma with intraoperatively diagnosed pleural seeding: Is main tumor resection beneficial for prognosis? J Thorac Cardiovasc Surg 2018;155:1238-49.e1.

4. Goldstraw P, Crowley J, Chansky K, et al. The IASLC Lung Cancer Staging Project: proposals for the revision of the TNM stage groupings in the forthcoming (seventh) edition of the TNM Classification of malignant tumours. J Thorac Oncol 2007;2:706-14.

5. Arrieta O, Escamilla-Lopez I, Lyra-Gonzalez I, et al. Radical aggressive treatment among non-small cell lung cancer patients with malignant pleural effusion without extra-thoracic disease. J Thorac Dis 2019;11:595-601.

6. Porcel JM. Malignant pleural effusions because of lung cancer. Curr Opin Pulm Med 2016;22:356-61.

7. Hsu JS, Han IT, Tsai TH, et al. Pleural Tags on CT Scans to Predict Visceral Pleural Invasion of Non-Small Cell Lung Cancer That Does Not Abut the Pleura. Radiology
2016;279:590-6.

8. Neri S, Menju T, Sowa T, et al. Prognostic impact of microscopic vessel invasion and visceral pleural invasion and their correlations with epithelial-mesenchymal transition, cancer stemness, and treatment failure in lung adenocarcinoma. Lung Cancer 2019;128:13-9.

9. Rami-Porta R, Bolejack V, Crowley J, et al. The IASLC Lung Cancer Staging Project: Proposals for the Revisions of the T Descriptors in the Forthcoming Eighth Edition of the TNM Classification for Lung Cancer. J Thorac Oncol 2015;10:990-1003.

10. Lakha S, Gomez JE, Flores RM, et al. Prognostic significance of visceral pleural involvement in early-stage lung cancer. Chest 2014;146:1619-26.

11. Jiwangga D, Cho S, Kim K, et al. Recurrence Pattern of Pathologic Stage I Lung Adenocarcinoma With Visceral Pleural Invasion. Ann Thorac Surg 2017;103:1126-31.

12. Okamoto T, Iwata T, Mizobuchi T, et al. Pulmonary resection for lung cancer with malignant pleural disease first detected at thoracotomy. Eur J Cardiothorac Surg 2012;41:25-30.

13. Ichinose $Y$, Tsuchiya R, Koike T, et al. The prognosis of patients with non-small cell lung cancer found to have carcinomatous pleuritis at thoracotomy. Surg Today 2000;30:1062-6.

14. Fukuse T, Hirata T, Tanaka F, et al. The prognostic significance of malignant pleural effusion at the time of thoracotomy in patients with non-small cell lung cancer. Lung Cancer 2001;34:75-81.

15. Mordant P, Arame A, Foucault C, et al. Surgery for metastatic pleural extension of non-small-cell lung cancer. Eur J Cardiothorac Surg 2011;40:1444-9.

16. Ichinose $Y$, Tsuchiya R, Koike T, et al. Prognosis of resected non-small cell lung cancer patients with carcinomatous pleuritis of minimal disease. Lung Cancer 2001;32:55-60.

17. Shiba M, Kakizawa K, Kohno H, et al. Prognostic implication of Ki-67 immunostaining in treating subclinical pleural cancer found at thoracotomy in lung cancer patients. Ann Thorac Surg 2001;71:1765-71.

18. Kodama K, Doi O, Higashiyama M, et al. Longterm results of postoperative intrathoracic chemothermotherapy for lung cancer with pleural dissemination. Cancer 1993;72:426-31.

19. Shigemura N, Akashi A, Ohta M, et al. Combined surgery of intrapleural perfusion hyperthermic chemotherapy and panpleuropneumonectomy for lung cancer with advanced pleural spread: a pilot study. Interact Cardiovasc Thorac 
Surg 2003;2:671-5.

20. Kimura M, Tojo T, Naito H, et al. Effects of a simple intraoperative intrathoracic hyperthermotherapy for lung cancer with malignant pleural effusion or dissemination. Interact Cardiovasc Thorac Surg 2010;10:568-71.

21. Skok K, Hladnik G, Grm A, et al. Malignant Pleural Effusion and Its Current Management: A Review. Medicina (Kaunas) 2019;55.

22. Rami-Porta R, Asamura H, Travis WD, et al. Lung cancer - major changes in the American Joint Committee on Cancer eighth edition cancer staging manual. CA Cancer J Clin 2017;67:138-55.

23. Fiorelli A, Santini M. In lung cancer patients where a malignant pleural effusion is found at operation could resection ever still be justified? Interact Cardiovasc Thorac
Surg 2013;17:407-12.

24. Fukui T, Yokoi K. The role of surgical intervention in lung cancer with carcinomatous pleuritis. J Thorac Dis 2016;8:S901-7.

25. Sequist LV, Yang JC, Yamamoto N, et al. Phase III study of afatinib or cisplatin plus pemetrexed in patients with metastatic lung adenocarcinoma with EGFR mutations. J Clin Oncol 2013;31:3327-34.

26. Ramalingam SS, Yang JC, Lee CK, et al. Osimertinib As First-Line Treatment of EGFR Mutation-Positive Advanced Non-Small-Cell Lung Cancer. J Clin Oncol 2018;36:841-9.

27. Ng KS, King Sun C, Boom Ting K, et al. Prognostic factors of EGFR-mutated metastatic adenocarcinoma of lung. Eur J Radiol 2020;123:108780.

Cite this article as: Li S, Yang X, Zhang S, Huang M, Ma Y, Yang Y. The prognostic analysis of lung cancer patients with occult malignant pleural disease at thoracotomy. Transl Cancer Res 2020;9(3):1689-1697. doi: 10.21037/tcr.2020.02.03 